\title{
DESIGN PARAMETERS FOR THE VERY LARGE HADRON COLLIDER
}

\author{
C. S. Mishra ${ }^{+}$and P.J. Limon, Fermilab ${ }^{*}$, Batavia, IL 60510
}

\begin{abstract}
The goal of the Very Large Hadron Collider (VLHC) is to extend the energy frontier beyond LHC. The proposed design center-of-mass energy for the VLHC pp collider is $100 \mathrm{TeV}$, with a luminosity of $1 \mathrm{e} 34 \mathrm{~cm}^{-2} \mathrm{sec}^{-1}$. At present accelerator designs and calculations are being carried out for two different magnet technologies, one using superferric magnets at 2 Tesla (T), the other using highfield design with $\mathrm{B}>10 \mathrm{~T}$. This paper will summarize the accelerator parameters for these two designs. We discuss the design parameters that have the largest effects on the performance of the accelerator and therefore need careful optimization.
\end{abstract}

\section{INTRODUCTION}

Hardon Colliders are the "discovery machines" for highenergy physics (HEP). The HEP and accelerator physics communities are working together to extend the energy frontier beyond LHC, and to understand the physics opportunities and the technical issues presented by the colliders.

A VLHC Steering Committee [1] was formed in response to the HEPAP subpanel Report [2] recommendation. The Steering Committee has representations from Fermilab, BNL, LBNL and Cornell University's Laboratory of Nuclear Studies and coordinates R\&D efforts in the United States.

The main technical issue is cost reduction. Considerable $R \& D$ is needed in accelerator technology, superconducting magnets, accelerator physics, and to reduce the overall cost of the accelerator construction and operation.

Initially, the U.S. site for the VLHC is assumed to be Fermilab. Hence, the injection chain comprise a $400 \mathrm{MeV}$ Linac, an $8.9 \mathrm{GeV}$ Booster, and the newly commissioned $150 \mathrm{GeV}$ Main Injector. The VLHC will have two new accelerators, a High Energy Booster (HEP) of 3 to $5 \mathrm{TeV}$ and a $50 \mathrm{TeV}$ per beam, pp collider.

\footnotetext{
+Email:mishra@fnal.gov

* Operated by the URA, under contract with the U.S. DOE.
}

Since the Indiana workshop in 1994 [3], work has been proceeding on the design parameters for the VLHC. During the Snowmass 96 meeting, the first set of comprehensive and self-consistent parameters was published [4]. Since that time we have modified several aspects of accelerator, resulting in a new set of parameters presented here.

\section{ACCELERATOR ENERGY}

The injection energy into the HEB is fixed near $150 \mathrm{GeV}$, because we want to use the Main Injector as an injector. Several discussions have taken place to use the Tevatron and inject at $1 \mathrm{TeV}$. However, the Tevatron requires 30 MW for its cryogenic plant. This high load for an injector that will be used for only a few moments each day seem excessive. Over the long run, the operating cost of the Tevatron is too high.

The VLHC magnet R\&D groups are investigating two different magnet technologies: high field (HF) (10-14 Tesla) [5-9] and low field (LF) (2 Tesla) [10]. The magnetic field quality at injection, eddy currents, persistent current and hysteric effects limit the ratio of energy at collision to injection for a synchrotron (SF). The Main Injector has a scale factor of 16.8, Tevatron 6, HERA 23, and LHC is designed to achieve 15.5. We have assumed this scale factor to be 20 for our primary accelerator design. This assumption needs study from the magnet, beam dynamics, and cost optimization points of view. For an HEB that is entirely on the Fermilab site, high-field magnets operating at $4 \mathrm{~K}$ could result in energy as high as $3-5 \mathrm{TeV}$. Operating injector at $1.8 \mathrm{k}$ is judged unreasonable due to cyclic energy loss. Obviously, a slightly larger machine could result in a lower vlhc SF at an expense of higher HEB SF. This problem could be eased by rebuilding a conventional accelerator, an MEB, in the existing Main Ring tunnel to increase the beam energy from $150 \mathrm{GeV}$ to $300 \mathrm{GeV}$. Such a machine could 
be quite inexpensive. For the low-field magnet, the HEB is limited by circumference of tunnel one is willing to build. A $3 \mathrm{TeV}$ low field HEB requires about $35 \mathrm{~km}$.

\section{LUMINOSITY}

The Fermilab accelerators have a minimum bunch spacing is $18.9 \mathrm{~ns}$. The expense of reducing the spacing and the detector triggering requirements encourages us to leave this the same. The design goal for the VLHC luminosity is $1 \times 10^{34} \mathrm{~cm}^{-2} \mathrm{sec}^{-1}$. This has been chosen due to of detector limitation, but could easily be a factor of 10 higher. At 1e34 luminosity a detector in VLHC will have less than 30 interactions per crossing. Table 1 gives the luminosity goals for the two VLHC options. It is important to note that the luminosity is not limited by proton economics, also there are several parameters that can be adjusted to achieve the same luminosity, for example smaller emittance and smaller bunch current. Smaller emittance in the HF option is due to the emittance damping caused by synchrotron radiation at colliding energy.

\section{$50 \mathrm{TeV} \quad 50 \mathrm{TeV}$ \\ Collider Collider \\ p p option p p option \\ (Low Field) (High Field)}

\begin{tabular}{|c|c|c|}
\hline Proton Per Bunch & $1.7 \mathrm{E}+10$ & $1.2 \mathrm{E}+10$ \\
\hline Number of Bunches & 100000 & 16000 \\
\hline $\begin{array}{l}\text { Revolution Frequency } \\
(\mathrm{kHz})\end{array}$ & 0.5 & 3.2 \\
\hline Beta Star at IP(m) & 0.1 & 0.1 \\
\hline Proton Emittance(95\%) & 15 & $8^{*}$ \\
\hline Form Factor & 0.48 & 0.48 \\
\hline $\begin{array}{l}\text { Typical Luminosity } \\
\left(\mathrm{cm}^{-2} \mathrm{sec}^{-1}\right)\end{array}$ & $1.1 \mathrm{E}+34$ & $1.1 \mathrm{E}+34$ \\
\hline $\begin{array}{l}\text { Integrated Luminosity } \\
\left(\mathrm{fb}^{-1} / \text { year }\right)\end{array}$ & 112 & 112 \\
\hline Interactions Per Crossing & 28 & 28 \\
\hline Bunch Spacing (ns) & 19 & 19 \\
\hline $\begin{array}{l}\text { Inelastic Cross Section } \\
(\mathrm{mb})\end{array}$ & 127 & 127 \\
\hline
\end{tabular}

Table 1 . The Luminosity goals of the VLHC at $100 \mathrm{TeV}$ center of mass. *HF option includes the effect of synchrotron radiation damping.

\section{MACHINE CIRCUMFERENCE \& BEAM PIPE RADIUS}

The circumference of the low field ( 2 Tesla) machine is $520 \mathrm{~km}$, whereas for high field (12 Tesla) machine it is 95 $\mathrm{km}$. The radius of the beam pipe for these machines is important because of beam instabilities. Smaller radius is desirable for cost reduction; magnet field errors, beam dynamics requirements, and alignment errors might lead to a larger radius. The proposed beam-pipe radius for the LF magnet is $9 \mathrm{~mm}$. The beam-pipe radius for HF magnet designs varies from 12 to $20 \mathrm{~mm}$. The beam pipe aperture is reduced from the physical magnet aperture by the thickness of the synchrotron radiation beam screen for the HF design.

The instability threshold for the transverse mode coupling instabilities (TMCI) is an issue for the LF design. This instability threshold is proportional to the cube of the beam pipe radius. It is expensive to increase the beam pipe radius and we are investigating several solutions to this instability.

The effect of ground motion, alignment, alignment drifts and vibration needs to be understood. Because of the large size of the ring in either design this could have implications on the correction system, when beam-pipe radius is small.

\section{LATTICE PARAMETERS}

Table 2 summarizes the lattice parameters for the two designs. The LF option uses combined function magnets. These magnets will have quadrupole and sextupole components. The phase advance per cell is chosen to be 60 degree. This reduces the requirement of large quadrupole strength and higher order correction schemes are easier to implement. These parameters are totally free and can be optimized, as the magnet data becomes available.

\begin{tabular}{|l|c|c|c|}
\hline Parameters & Units & Low-Field & High-Field \\
\hline $\begin{array}{c}1 / 2 \text { Cell } \\
\text { Length }\end{array}$ & M & 122 & 260 \\
\hline $\begin{array}{l}\text { Phase } \\
\text { Advance } \\
\text { per cell }\end{array}$ & Degree & 60 & 60 \\
\hline $\begin{array}{l}\text { Average } \\
\text { Beta }\end{array}$ & M & 130 & 600 \\
\hline $\begin{array}{l}\text { Max } \\
\text { Dispersion }\end{array}$ & M & 5 & 23 \\
\hline
\end{tabular}

Table 2. VLHC lattice parameters.

\section{RF PARAMETERS}

The frequency of the HEB and Collider must be a multiple of $53 \mathrm{MHz}$, which will make synchronization and injection easier. The VLHC will most likely use superconducting RF for acceleration although there is no serious problem using the normal conducting RF. Some of the RF parameters of general interest for superconducting RF system being discussed for the VLHC are summarized in Table 3 [11]. A total voltage of $200 \mathrm{MV}$ for the Low field option and $40 \mathrm{MV}$ for the high field option have 
been chosen to keep the acceleration time to less than 15 minutes.

\begin{tabular}{|c|c|c|}
\hline Parameters & Units & Value \\
\hline $\mathrm{f}_{\mathrm{RF}}$ & $\mathrm{MHz}$ & 478 \\
\hline Operating temp & $\mathrm{K}$ & 4.2 \\
\hline $\mathrm{E}_{\text {acc }}$ & $\mathrm{MV} / \mathrm{m}$ & 6 \\
\hline Volts/Cavity & $\mathrm{MV}$ & 7.5 \\
\hline Length of Bucket & $\mathrm{Cm}$ & 21 \\
\hline $\begin{array}{c}\text { No of cavities for } \\
\text { 200 MV LF } \\
\text { injection }\end{array}$ & 27 \\
\hline $\begin{array}{c}\text { No. of cavity for } \\
\text { 40 MV HF } \\
\text { injection }\end{array}$ & \\
\hline
\end{tabular}

Table 3. VLHC Superconducting RF parameters.

Finally the longitudinal parameters of the VLHC are summarized in Table 4. Most of these parameters can be optimized as design progress. The longitudinal emittance and bunch length effects the stabilities of the machine.

\begin{tabular}{|c|c|c|c|}
\hline Parameter & Units & LF & HF \\
\hline $\begin{array}{c}\text { Long. Emitt } \\
\text { time }\end{array}$ & $\mathrm{eV.sec}$ & 2 & 0.3 \\
\hline $\begin{array}{c}\text { Long Damping } \\
\text { RF freq }\end{array}$ & $\mathrm{MHz}$ & 478 & 1.3 \\
\hline $\begin{array}{c}\text { RF harmonic } \\
\text { number }\end{array}$ & $\mathrm{eV}-\mathrm{sec}$ & 18 & 478 \\
\hline Bucket Area & $0.2 / 5$ & $1 / 14$ \\
\hline $\begin{array}{c}\text { Synch tune } \\
\text { (coll/inj) }\end{array}$ & $\mathrm{Cm}$ & $7.6(5.5)$ & $5.6(7.2)$ \\
\hline $\begin{array}{c}\text { Bunch Length } \\
\text { (coll/inj) }\end{array}$ & & & \\
\hline
\end{tabular}

Table 4. Longitudinal parameters for the VLHC.

\section{SUMMARY}

This paper gives a snapshot of some of the parameters being used in the accelerator design of the low field and high field VLHC. Collaborative efforts between magnet builders, accelerator technology developers and accelerator physicists throughout the process of accelerator design will be used to refine these parameters.

\section{REFERENCES}

[1] The VLHC Steering Committee website http://vlhc.org.

[2] HEPAP Subpanel on "Planning for the Future of U.S. High Energy Physics, February 1998. (Gilman Panel).

[3] Proceedings of the Workshop on Future Hadron Collider Facilities in the US, Bloomington 19994, Fermilab TM-1907.
[4] G. Dugan, P. J. Limon and M. Syphers, "Really Larger Hadron Collider Working Group Summary", Proceeding of DPF/DPB Snowmass 96 summer study.

[5] T. ARCAN et al., "Conceptual Design of the Fermilab Nb3Sn High Field Dipole Model", Proceedings of PAC99.

[6] K. Chow et al., "Fabrication and Test Results of a Prototype, Nb3Sn Superconducting Racetrack Dipole Magnet", Proceedings of PAC99.

[7] R. Gupta, "Field Quality in a Common Coil Design Magnet System", Proceedings of PAC99.

[8] S. Caspi et al., "Mechanical Design of a High Field Common Coil Magnet", Proceedings of PAC99

[9] A.K. Ghosh et al., "A Common Coil Magnet for Testing High Field Superconductors”, Proceedings of PAC99.

[10] G. W. Foster et al., "Measurements of a Crenellated Iron Pole Tip For The VLHC Transmission Line Magnet", Proceedings of PAC99.

[11] L. Doolittle, "RF Choice for the VLHC", VLHC Accelerator Physics workshop, TJNAF, Feb 8-11, 1999. 